\title{
Fault Modeling and Functional Test Methods for Digital Microfluidic Biochips
}

\author{
Tao Xu, Student Member, IEEE, and Krishnendu Chakrabarty, Fellow, IEEE
}

\begin{abstract}
Dependability is an important attribute for microfluidic biochips that are used for safety-critical applications, such as point-of-care health assessment, air-quality monitoring, and food-safety testing. Therefore, these devices must be adequately tested after manufacture and during bioassay operations. Known techniques for biochip testing are all function oblivious (i.e., while they can detect and locate defect sites on a microfluidic array, they cannot be used to ensure correct operation of functional units). In this paper, we introduce the concept of functional testing of microfluidic biochips. We address fundamental biochip operations, such as droplet dispensing, droplet transportation, mixing, splitting, and capacitive sensing. Long electrode actuation times are avoided to ensure that there is no electrode degradation during testing. The functional testing of pin-constrained biochips is also studied. We evaluate the proposed test methods using simulations as well as experiments for a fabricated biochip.
\end{abstract}

Index Terms-Dependability, lab-on-chip, microfluidics, reconfiguration, testing.

\section{INTRODUCTION}

$\mathbf{M}$ ICROFLUIDICS-BASED biochips, also referred to as lab-on-a-chip, are replacing cumbersome and expensive laboratory equipment for applications, such as cell high-throughput sequencing, parallel immunoassays, protein crystallization, blood chemistry for clinical diagnostics, environmental toxicity monitoring, and cell biology [1]-[7]. These miniaturized and automated biochip devices offer the advantages of higher sensitivity, lower cost due to smaller sample and reagent volumes, higher levels of system integration, and less likelihood of human error.

A popular class of microfluidic biochips is based on continuous fluid flow in permanently etched microchannels. These devices rely on either micropumps or microvalves to electrical methods based on electrokinetics to control continuous sample flows [4], [6]-[8]. Specific to electroosmosis flows, a metal-oxide semiconductor field-effect transistor (MOSFET)-like design, referred to as a FlowFET, has also been proposed [9].

Manuscript received September 16, 2008; revised January 19, 2009. Current version published July 29,2009 . This work was supported in part by the National Science Foundation under Grant CCF-0541055. A preliminary version of this paper was published in Proc. International Test Conference, 2007. This paper was recommended by Associate Editor T. Le.

T. Xu is with Cisco Systems, Inc., Morrisville, NC 27560 USA (e-mail: txu2@cisco.com).

K. Chakrabarty is with the Department of Electrical and Computer Engineering, Duke University, Durham, NC 27708 USA (e-mail: krish@ee.duke. edu).

Color versions of one or more of the figures in this paper are available online at http://ieeexplore.ieee.org.

Digital Object Identifier 10.1109/TBCAS.2009.2022173
An alternative category of microfluidic biochips relies on "digital microfluidics," which is based on the principle of electrowetting on dielectric [3]. By manipulating discrete droplets of the nanoliter volume by using a patterned array of electrodes, miniaturized bioassay protocols (in terms of liquid volumes and assay times) are mapped and executed on a microfluidic chip. Therefore, digital microfluidic biochips require only nanoliter volumes of samples and reagents. The electrodes in the microfluidic array are controlled by independent control pins. This allows for free movement of the droplets on the array. Digital microfluidic biochips offer continuous sampling and analysis capabilities for online and real-time chemical/biological sensing. These systems also have a desirable property referred to as dynamic reconfigurability, where microfluidic modules can be relocated to other places on the electrode array, without affecting functionality, during the concurrent execution of a set of bioassays [10]. Reconfigurability enables microfluidic biochips to be "adaptive" for a wide variety of applications. System reconfiguration can also be used to bypass the faulty cells to enable microfluidic arrays to provide reliable results even in the presence of defects.

Recent years have seen a steady increase in the level of integration and system complexity of digital microfluidic biochips [1], [6], [11], [12]. A prototype has been developed for gene sequencing through synthesis [1], which targets the simultaneous execution of $10^{6}$ fluidic operations and the processing of billions of droplets. Reference [6] proposed an integrated microfluidic platform that can carry out RNA extraction and DNA synthesis on a single chip. Other biochip systems are being designed for protein crystallization, which requires the concurrent execution of hundreds of operations [11]. A commercially available droplet-based (using dielectrophoresis) biochip embeds more than $60000020-\mu \mathrm{m} \times 20-\mu \mathrm{m}$ electrodes with integrated optical detectors [12].

As in the case of integrated circuits (ICs), an increase in the density and area of microfluidics-based biochips will lead to high defect densities, thereby reducing yield, especially for newer technologies. However, dependability is an important system attribute for biochips. Dependability is essential for safety-critical applications, such as point-of care diagnostics, health assessment, and screening for infectious diseases, air-quality monitoring, and food-safety tests, as well as for pharmacological procedures for drug design and discovery that require high precision levels. Some manufacturing defects may be latent, and they may produce errors during field operation. In addition, harsh operational environments and biological samples (e.g., proteins) may introduce physical defects, such as particle contamination and residue on surfaces due to adsorption. 
Since complicated fluidic operations are repeatedly executed with high precision in compact microfluidic arrays, a group of cells is repeatedly required to perform a large number of operations. Traditional structural test methods, which use test droplets to traverse the target array, do not provide a sufficient level of confidence that these fluidic operations can be reliably performed on the array [13]. For instance, some unit cells (i.e., electrodes) may function correctly during droplet transportation, but they might malfunction during droplet dispensing from reservoirs. Likewise, unit cells that can be reliably combined to operate as a mixer may malfunction when they are used for droplet splitting. Moreover, a structural test does not cover nonreconfigurable modules, such as capacitive sensing circuits. A defect involving any of the modules may result in catastrophic failure during bioassay execution. Therefore, before we use synthesis methods to map bioassay protocols to a microfluidic array [14], it is important to carry out functional testing to verify the integrity of the underlying microfluidic platform. To ensure that manufactured biochips are competitive in the emerging low-cost market for disposable biochips and to avoid electrode degradation resulting from excessive actuation, test methodologies should be inexpensive, quick, and effective.

In this paper, we first present various defects that are typical for digital microfluidic biochips. We relate these defects to logical fault models that can be viewed not only in terms of traditional shorts and opens, but which also target biochip functionality. Based on these fault models, we introduce the idea of functional testing of digital microfluidic modules. We develop cost-effective functional test methods that target fluidic operations, such as droplet dispensing, droplet transportation, mixing, and splitting. These methods also test the functionality of nonreconfigurable modules, such as capacitive-sensing circuits. The proposed methods allow functional testing by using parallel droplet pathways in online and offline scenarios. For each function, the proposed approach identifies "qualified regions" (i.e., groups of cells that pass the test). Instead of placing fluidic modules in a fault-oblivious manner on the microfluidic array, synthesis tools can map modules only to qualified regions. In this way, the reliability of the synthesized biochip is significantly increased. The application of these methods to pin-constrained biochips is also discussed. We evaluate the proposed functional test methods using simulations as well as experiments for a fabricated biochip.

The rest of this paper is organized as follows. Section II provides an overview of digital microfluidic biochips. Section III reviews different methodologies for pin-constrained biochips design. In Section IV, we discuss prior work on defect modeling and structural testing. In Section V, we relate defects to fault models and observable errors, and we list various fault models for digital microfluidic biochips. We also introduce the concept of functional testing and propose effective methods to test the biochip for droplet dispensing, droplet transportation, mixing, splitting, and capacitive sensing. Section VI discusses functional testing for pin-constrained chips. In Section VII, we apply these techniques to a fabricated chip and present simulation results. Finally, conclusions are drawn in Section VIII.

\section{Digital Microfluidic BiochiPS}

A digital microfluidic biochip utilizes the phenomenon of electrowetting to manipulate and move nanoliter droplets con-

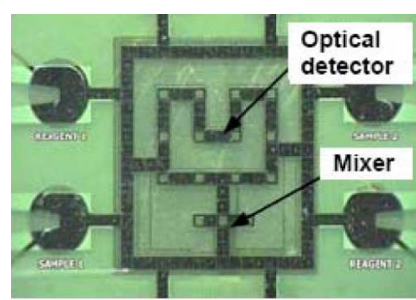

Glass-substrate plateform

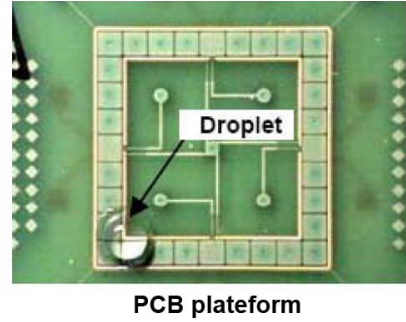

PCB plateform
Fig. 1. Fabricated digital microfluidic arrays [15].

taining biological samples on a 2-D electrode array [3]. A unit cell in the array includes a pair of electrodes that acts as two parallel plates. The bottom plate contains a patterned array of individually controlled electrodes, and the top plate is coated with a continuous ground electrode. A droplet rests on a hydrophobic surface over an electrode, as shown in Fig. 1. It is moved by applying a control voltage to an electrode adjacent to the droplet and, at the same time, deactivating the electrode just under the droplet. This electronic method of wettability control creates interfacial tension gradients that move the droplets to the charged electrode. Using the electrowetting phenomenon, droplets can be moved to any location on a 2-D array.

By varying the patterns of control voltage activation, many fluid-handling operations, such as droplet merging, splitting, mixing, and dispensing can be easily executed. For example, mixing can be performed by routing two droplets to the same location and then turning them about some pivot points.

An alternative coplanar design was recently proposed in [15]. It eliminated the need for a conductive top plate by implementing coplanar ground electrodes either in the same conductive layer as the electrodes. The coplanar design provided a viable solution for an inexpensive and fast turnaround process to fabricate digital microfluidic systems.

The digital microfluidic platform offers the additional advantage of flexibility, referred to as dynamic reconfigurability, since fluidic operations can be performed anywhere on the array. Droplet routes and the operation scheduling result are programmed into a microcontroller that drives electrodes in the array. In addition to electrodes, optical detectors, such as LEDs and photodiodes, are also integrated in digital microfluidic arrays to monitor colorimetric bioassays [5].

\section{Pin-Constrained ChiP Design}

As discussed in Section I, each electrode in the microfluidic array is connected to a dedicated control pin; it can therefore be activated independently. This control scheme is referred to as direct addressing. This method provides the maximum freedom for droplet manipulation, but it requires an excessive number of control pins. For example, a total of $10^{4}$ pins are needed to independently control the electrodes in a $100 \times 100$ array. Multilayer electrical connection structures and wire-routing solutions are complicated by the large number of independent control pins in these arrays. Product cost, however, is a major marketability driver due to the one-time-use (disposable) nature of most emerging devices. Thus, the design of pin-constrained digital microfluidic arrays is of considerable importance for the emerging marketplace. 
Pin-constrained design of digital microfluidic biochips was first proposed and analyzed in [16]. The number of control pins for a fabricated electrowetting-based biochip is minimized by using a multiphase bus for the fluidic pathways. Every $n$th electrode in an $n$-phase bus is electrically connected. Thus, only $n$ control pins are needed for a transport bus, irrespective of the number of electrodes that it contains. The multiphase bus method is useful for a 1-D (linear) array.

An alternative method based on a cross-reference driving scheme is presented in [17]. The electrode rows are patterned on the top and bottom plates, and placed orthogonally. In order to drive a droplet along the $\mathrm{X}$-direction, electrode rows on the bottom plate serve as driving electrodes, while electrode rows on the top serve as reference ground electrodes. The roles are reversed for movement along the Y-direction. This method allows control of an $N \times M$ grid array with only $N+M$ control pins.

Another pin-constrained design method is based on the partitioning of the microfluidic array and the assignment of a small number of control pins to a large number of electrodes in each partition. The partitioning algorithm is based on the concept of "droplet trace," which is extracted from the scheduling and droplet routing results produced by a synthesis tool [18]. The key idea is to "virtually" partition the array into regions. At any given time, partitions use nonoverlapping sets of pins.

More recently a broadcast-addressing-based design technique for pin-constrained multifunctional biochips has been proposed [19]. This method provides high throughput for bioassays and it reduces the number of control pins by identifying and connecting control pins with "compatible" actuation sequences. In this paper, we investigate the application of the proposed functional test method to biochip prototypes designed by using these schemes.

\section{RELATED PRIOR WORK ON BIOCHIP TESTING}

The testing of microfluidic biochips has recently been investigated. These test methods add fluid-handling aspects to MEMS testing techniques [20]-[22]. Test methods have been proposed for continuous-flow and digital microfluidic biochips. An excellent review is available in [23]. A fault model and a fault simulation method for continuous-flow microfluidic biochips have been proposed in [13]. For digital microfluidic chips, techniques for defect classification, test planning, and test-resource optimization have been presented [13]. Defect classification methods are discussed in [13] and corresponding test procedures are described in [24]. Defects have been classified as being either catastrophic or parametric, and techniques have been developed to detect these defects by electrostatically controlling and tracking droplet motion.

The work in [13] and [24] facilitates concurrent testing, which allows fault detection and biomedical assays to run simultaneously on a microfluidic system [25]. A drawback of [13], however, is that it does not present any automated techniques for optimizing the test application procedure. Reference [26] first proposed a test planning and test-resource optimization method. The test planning problem is mapped to the Hamilton cycle problem from graph theory. An alternative method based on Euler paths is proposed in [27]. This method maps a digital microfluidic biochip to an undirected graph and a test droplet is routed along the Euler path derived from the graph to pass through all of the cells in the array. Fault diagnosis is carried out by using multiple test application steps and adaptive Euler paths.

A drawback of the aforementioned "structural" test methods is that they focus only on fault modeling, and the test and diagnosis of physical defects, and they overlook module functionality. Therefore, these methods can only guarantee that a biochip is defect free. However, a defect-free microfluidic array can also malfunction in many ways. For example, a defect-free reservoir may result in large volume variations when droplets are dispensed from it. A splitter composed of three defect-free electrodes may split a big droplet into two droplets with significantly unbalanced volumes. These phenomena, referred to as malfunctions, are not the result of electrode defects. Instead, they are activated only for certain patterns of droplet movement or fluidic operations. These malfunctions can have serious consequences on the integrity of bioassay results. Therefore, to ensure robust execution of the target bioassay, we must carry out more comprehensive test procedures, which not only target defective cells, but also lead to the detection of malfunctioning microfluidic modules.

\section{Functional Testing}

In this section, we propose a comprehensive test procedure, referred to as functional testing, which targets the functional operation of microfluidic modules. To avoid ambiguity, we refer to the test methods discussed in [13], [24], and [25] as a structural test, since they route test droplets to all of the electrodes in the array to ensure structural integrity. The structural test targets physical defects, which are defined as the underlying cause of erroneous chip operation, where the defect affects either a unit cell (electrode) or the electrical connection to the unit cell. Functional testing, on the other hand, involves test procedures to check whether groups of cells can be used to perform certain operations (e.g., droplet mixing and splitting). For the test of a specific operation, the corresponding patterns of droplet movement are carried out on the target cluster of cells. If a target cell cluster fails, the test (e.g., the mixing test) is labeled as a malfunctioning cluster, which implies that the synthesis tool cannot place the corresponding module - a mixer in this case-in this region.

As in the case of structural testing, we first develop a fault model for functional testing. Since functional testing is an extension of structural testing, all of the defect-oriented fault models are also included here. Therefore, we start from the fault models proposed in [13]. Malfunctions in fluidic operations are then identified and added to the list. In this way, we derive a more comprehensive set of fault models, as shown in Table I.

Next, we propose efficient functional test methods to detect the defects and malfunctions listed in Table I. The dispensing test, mixing test, splitting test, and capacitive sensing test are developed to address the corresponding malfunctions. A routing test procedure is used to detect all physical defects. 
TABLE I

FAult Models for Digital Microfluidic Biochips

\begin{tabular}{|l|l|c|l|l|}
\hline $\begin{array}{l}\text { Cause of } \\
\text { defect }\end{array}$ & $\begin{array}{l}\text { Defect } \\
\text { type }\end{array}$ & $\begin{array}{l}\text { Number } \\
\text { of } \text { cells }\end{array}$ & $\begin{array}{l}\text { Fault } \\
\text { model }\end{array}$ & $\begin{array}{l}\text { Observable } \\
\text { error }\end{array}$ \\
\hline $\begin{array}{l}\text { Excessive actuation } \\
\text { voltage applied to an electrode }\end{array}$ & Dielectric breakdown & 1 & $\begin{array}{l}\text { Dropletelectrode short (a } \\
\text { short between the droplet and } \\
\text { the electrode) }\end{array}$ & $\begin{array}{l}\text { Droplet undergoes electrolysis, which } \\
\text { prevents its further transportation }\end{array}$ \\
\hline $\begin{array}{l}\text { Electrode actuation for } \\
\text { excessive duration }\end{array}$ & $\begin{array}{l}\text { Irreversible charge concentration } \\
\text { on an electrode }\end{array}$ & 1 & $\begin{array}{l}\text { Electrode-stuck-on (the } \\
\text { electrode remains constantly } \\
\text { activated) }\end{array}$ & $\begin{array}{l}\text { Unintentional droplet operations or } \\
\text { stuck droplets }\end{array}$ \\
\hline $\begin{array}{l}\text { Excessive mechanical force } \\
\text { applied to the chip }\end{array}$ & $\begin{array}{l}\text { Misalignment of parallel plates } \\
\text { (electrodes and ground plane) }\end{array}$ & 1 & $\begin{array}{l}\text { Pressure gradient (net static } \\
\text { pressure in some direction) }\end{array}$ & $\begin{array}{l}\text { Droplet transportation without } \\
\text { activation voltage }\end{array}$ \\
\hline Coating failure & Non-uniform dielectric layer & 1 & $\begin{array}{l}\text { Dielectric islands } \\
\text { (islands of Teflon coating) }\end{array}$ & $\begin{array}{l}\text { Fragmentation of droplets and their } \\
\text { motion is prevented }\end{array}$ \\
\hline $\begin{array}{l}\text { Abnormal } \\
\text { metal layer } \\
\text { deposition } \\
\text { and etch } \\
\text { variation } \\
\text { during } \\
\text { fabrication }\end{array}$ & Grounding Failure & 1 & $\begin{array}{l}\text { Floating droplets (droplet are } \\
\text { not anchored })\end{array}$ & Failure of droplet transportation \\
\cline { 2 - 4 } & Broken wire to control source & 1 & $\begin{array}{l}\text { Electrode open (electrode } \\
\text { actuation is not possible) }\end{array}$ & $\begin{array}{l}\text { Failure to activate the } \\
\text { electrode for droplet } \\
\text { transportation }\end{array}$ \\
\hline $\begin{array}{l}\text { Particle contamination or } \\
\text { liquid residue }\end{array}$ & $\begin{array}{l}\text { Metal connection between two } \\
\text { adjacent electrodes }\end{array}$ & 2 & $\begin{array}{l}\text { Electrode short (short } \\
\text { between electrodes) }\end{array}$ & $\begin{array}{l}\text { A droplet resides in } \\
\text { the middle of the two shorted } \\
\text { electrodes, and its transport along one } \\
\text { or more directions } \\
\text { cannot be achieved }\end{array}$ \\
\hline $\begin{array}{l}\text { Protein adsorption during } \\
\text { bioassay [28] }\end{array}$ & $\begin{array}{l}\text { Sample residue on electrode } \\
\text { surface }\end{array}$ & 1 & Resistive open at electrode & Droplet transportation is impeded. \\
\cline { 2 - 4 } & Contamination & $\begin{array}{l}\text { Assay results are outside the range of } \\
\text { possible outcomes }\end{array}$ \\
\hline
\end{tabular}

\begin{tabular}{|l|l|c|l|l|}
\hline $\begin{array}{l}\text { Cause of } \\
\text { malfunction }\end{array}$ & $\begin{array}{l}\text { Malfunction } \\
\text { type }\end{array}$ & $\begin{array}{l}\text { Number } \\
\text { of } \text { cells }\end{array}$ & $\begin{array}{l}\text { Fault } \\
\text { model }\end{array}$ & $\begin{array}{l}\text { Observable } \\
\text { error }\end{array}$ \\
\hline $\begin{array}{l}\text { Electrode actuation for } \\
\text { excessive duration }\end{array}$ & $\begin{array}{l}\text { Irreversible charge } \\
\text { concentration on the dispensing } \\
\text { electrode }\end{array}$ & 3 & $\begin{array}{l}\text { Dispensing-stuck-on (droplet } \\
\text { is dispensed by not fully cut } \\
\text { off from the reservoir) }\end{array}$ & $\begin{array}{l}\text { No droplet can be dispensed from the } \\
\text { reservoir }\end{array}$ \\
\hline $\begin{array}{l}\text { Electrode shape variation in } \\
\text { fabrication }\end{array}$ & Deformity of electrodes & 3 & $\begin{array}{l}\text { No overlap between droplets } \\
\text { to be mixed and center } \\
\text { electrode }\end{array}$ & Mixing failure \\
\hline $\begin{array}{l}\text { Electrode electrostatic } \\
\text { property variation in } \\
\text { fabrication }\end{array}$ & Unequal actuation voltages & 3 & $\begin{array}{l}\text { Pressure gradient (net static } \\
\text { pressure in some direction) }\end{array}$ & Unbalanced volumes of split droplets \\
\hline Bad soldering & $\begin{array}{l}\text { Parasitic capacitance in the } \\
\text { capacitive sensing circuit }\end{array}$ & 1 & $\begin{array}{l}\text { Oversensitive or insensitive } \\
\text { capacitive sensing }\end{array}$ & False positive/negative in detection \\
\hline
\end{tabular}

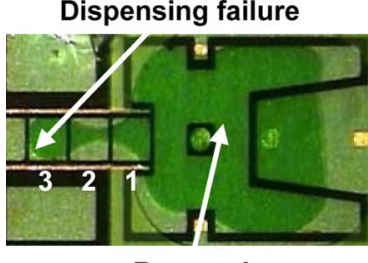

Reservoir

(a)

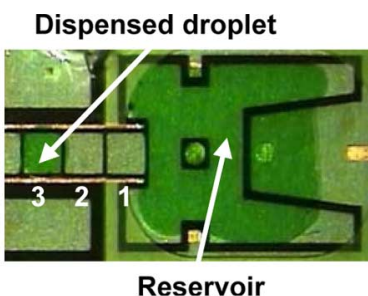

(b)
Fig. 2. Illustration of (a) normal dispensing and (b) dispensing failure for a fabricated microfluidic biochip.

\section{A. Dispensing Test}

The dispensing test targets the malfunctioning of the dispensing operation. Fig. 2 provides a comparison between normal dispensing and an example of dispensing failure. As shown in Fig. 2(b), the dispensed droplet in a malfunctioning scenario cannot be detached from the droplet in the reservoir. Therefore, when we move the dispensed droplet away from the reservoir, an additional droplet from the reservoir is extracted and moved as well.

In this case, the dispensed "droplet" can be several times larger than the normal size, which may result in the catastrophic failure of a volume-sensitive bioassay.
Here, we propose a test method based on capacitive sensing to detect these dispensing failures. The circuit diagram for fault detection is shown in Fig. 3. It has been shown in the literature that dispensing involves a reservoir and the three electrodes that are closest to it [30]. We therefore define every reservoir together with its three neighbor electrodes as a dispensing cluster. The third electrode in the cluster (i.e., the electrode farthest from the reservoir) is connected to a capacitive-sensing circuit for the test readout, see Fig. 3. When the test starts, a droplet is dispensed from the reservoir until it reaches the third electrode. We expect a positive pulse with a normal amplitude for fault-free dispensing and dispensing failure. Next, we route the dispensed droplet one electrode in the forward direction. During correct dispensing, the fully dispensed droplet moves completely to the fourth electrode; therefore, no pulse is detected by the capacitive sensing circuit output at this time. However, if the droplet undergoes a dispensing failure and it is still connected to the liquid in the reservoir, there must be some fluid left at the third electrode, which is indicated by a positive pulse, with smaller amplitude in the test readout. Therefore, we can easily detect a dispensing failure by reading the output of the capacitive sensing circuit, as shown in Fig. 4.

To identify abnormal droplets, two threshold values for the pulse amplitude are used. These thresholds are determined through calibration of the sensing circuit. First, we fix a nom- 


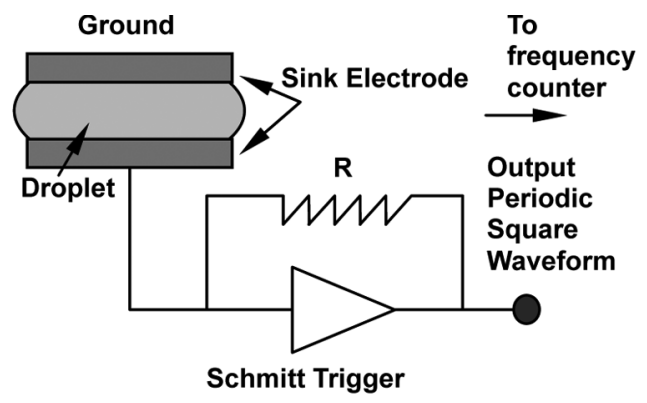

(a)

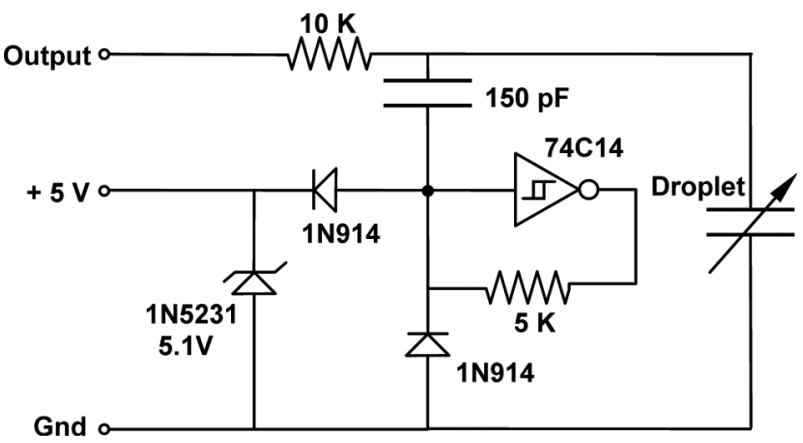

(b)

Fig. 3. Capacitive-sensing circuit. (a) Outline [29]. (b) Detailed circuit [29].

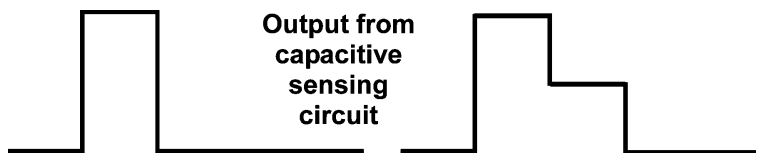

Fig. 4. Test readouts for (a) normal dispensing and (b) dispensing failure.

inal value $\mu$ and a maximum allowable droplet volume variance $\sigma$. Then, two droplets with a volume of $\mu+\sigma$ and $\mu-\sigma$ are routed to the sensing circuit. Signal levels are recorded and used as the upper and lower threshold values, respectively.

\section{B. Routing Test and Capacitive Sensing Test}

Routing test focuses on evaluating a single electrode's ability to transport droplets. This procedure is similar to that proposed earlier for structural test [13], [24], [25]. In the structural test, a test droplet is dispensed and routed to cross the target electrode from two orthogonal directions (i.e., along the row and the column directions). The routing problem can be solved by mapping the array to an undirected graph and applying the Eulerpath-based method [27] as shown in Fig. 5. On the other hand, a test droplet must be routed along all four directions relative to the target electrode. We can solve the route planning problem in this case by mapping the target array to a directed graph, which can be easily derived by replacing every edge in the undirected graph with two directed edges in the opposite directions. The Euler-path-based method is then applied to the directed graph to derive a test plan for the routing test.

Note that in the structural test, a test droplet is routed to traverse the array, following the Euler path derived from the undirected graph in Fig. 5(a). As a result of this procedure, half of the directed edges in Fig. 5(b) are also traversed, with exactly one edge visited for each pair of directed edges between two nodes. The edges that are not visited can be ordered to form a "reverse

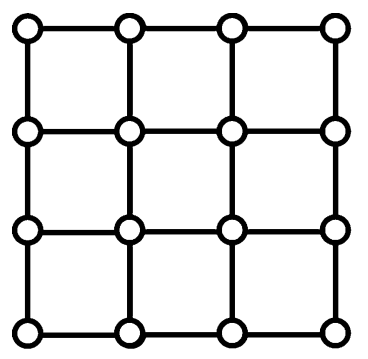

(a)

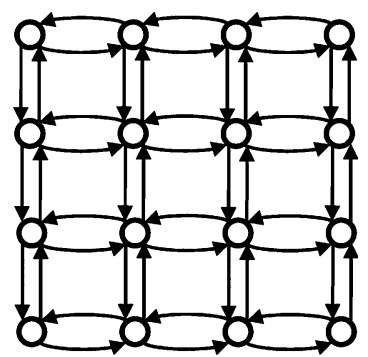

(b)
Fig. 5. (a) Undirected graph for the Euler-path-based structural test. (b) The corresponding directed graph for the Euler-path-based routing test.

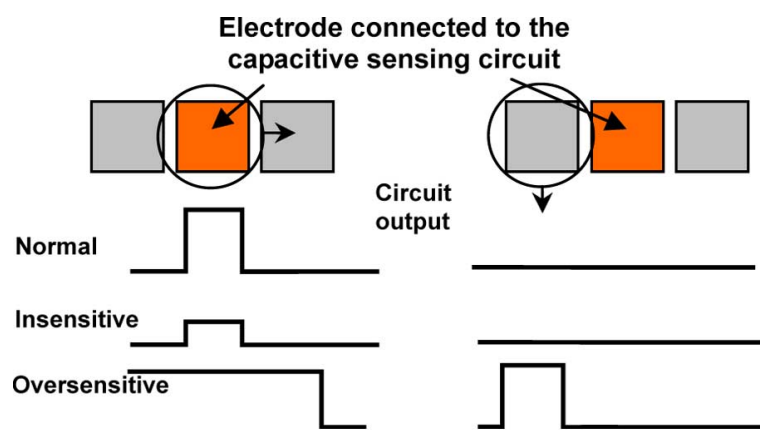

(a)

(b)

path" corresponding to the Euler path derived before. Therefore, the routing test can be carried out by applying two iterations of the structural test in opposite directions. Recall that all of the defects listed in Table I can be detected by the structural test; therefore, they are also detected by the aforementioned routing test procedure.

The aforementioned test procedure also tests the functionality of the capacitive-sensing circuit. If a test droplet is routed to visit the electrode connected to the target capacitive sensing circuit, a positive pulse is expected at the output of the sensing circuit. By examining the amplitude of the positive pulse, we can determine whether a capacitive sensing circuit is normal, insensitive, or oversensitive, as shown in Fig. 6.

\section{Mixing and Splitting Test}

Next, we discuss the functional testing of two widely used microfluidic modules - mixers and splitters. In a digital microfluidic biochip, two droplets are mixed within a cluster of electrodes, referred to as the mixer. Even though mixer designs and configurations vary considerably [31], [32], the underlining mixing mechanisms remain the same for all designs and configurations. Two droplets are merged at one electrode and routed to move about some pivots in the mixer, as shown in Fig. 7.

Thus, a mixing functional test is equivalent to the testing of the merging and routing operations within the target cell cluster. Recall that the droplet routing test has been addressed in Section V-B; therefore, a mixing test can be reduced to a droplet merging test, which checks a series of three adjacent electrodes to determine whether two droplets can be merged on them. For a microfluidic array, a simple test method carries out droplet merging on every group of three adjacent electrodes, 


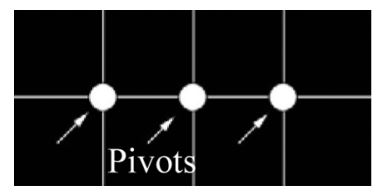

(a)

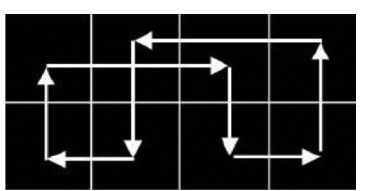

(b)
Fig. 7. (a) Pivots and (b) routing path for a $2 \times 4$ microfluidic mixer.

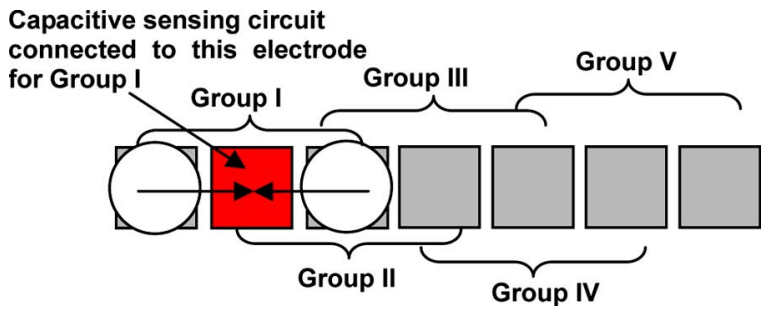

Fig. 8. Example of merging test.
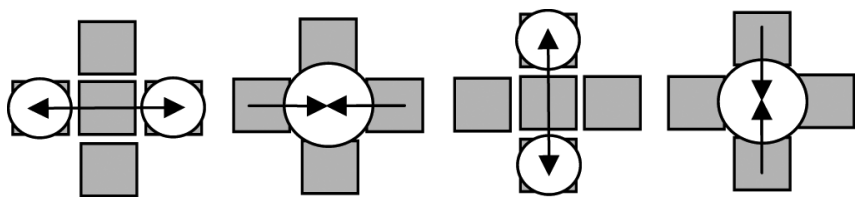

Fig. 9. Mixing and splitting test for all of the groups of three electrodes that are centered on a given electrode.

one at a time. For this three-electrode test, the test outcome is read out by using a capacitive-sensing circuit connected to the center electrode, on which droplets are supposed to be merged, as shown in Fig. 8. However, since every electrode can be the center of a set of three electrodes, we have to connect a capacitive-sensing circuit to it, which results in high production cost. Moreover, the serial processing method requires a large number of droplet manipulation steps and electrode actuations. As shown in Table I, excessive actuation may result in a variety of catastrophic defects. Therefore, efficient algorithms are needed for the droplet-merging test.

Droplet splitting is simpler compared to mixing. The fluidic operation involves three adjacent electrodes. By applying an appropriate electrode-actuation sequence, a droplet that rests on the center electrode is split into two smaller droplets, which rest on the two side electrodes. Thus, a split operation can be viewed as the reverse of droplet merging. Consequently, splitting test can be carried out by applying the merging test methods in a reverse manner. The only difference lies in the fact that instead of connecting a capacitive-sensing circuit to the center electrode, the splitting test attaches two capacitive-sensing circuits to the two side electrodes. The test outcome is evaluated by comparing output amplitudes of the two sensing circuits.

We next combine these two tests into a unified test application procedure. We start from the simple case where the mixing and splitting test are carried out for two three-electrode groups centered at one electrode. For simplicity, we limit our discussion to linear merging and splitting (i.e., the electrodes involved are linearly aligned in the same row/column). The test procedure is illustrated in Fig. 9.

In Fig. 9, we carry out the mixing and splitting test using four steps (i.e., horizontal splitting, horizontal mixing, vertical

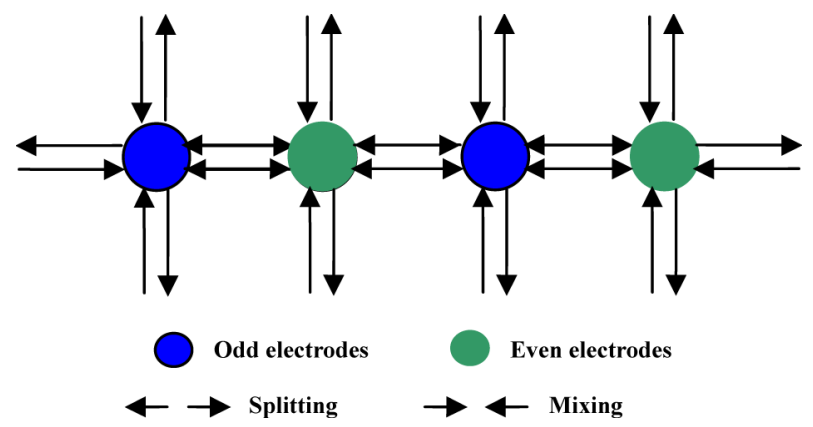

Fig. 10. Parallel mixing and splitting test for a row of electrodes.

splitting, and vertical mixing). Note that the ordering is carefully chosen such that the four steps can be carried out consecutively, without additional routing steps needed in between. However, this procedure still requires every electrode to be connected to a capacitive-sensing circuit. Moreover, in order to extend this test scheme to a microfluidic array, we need $4 N^{2}$ manipulation steps for an $N \times N$ array of electrodes, which is very inefficient.

To achieve higher test efficiency and lower hardware cost, we apply the single-electrode test methods in parallel for array testing. The key idea is to carry out mixing and splitting test for all of the electrodes in a row/column concurrently. For simplicity of analysis, we demonstrate the method using a directed graph, where each electrode is mapped to a node in the graph, and each mixing or splitting test step is represented by a pair of directed edges; see Fig. 10.

The electrodes in Fig. 10 are labeled as being either "even" or "odd." We carry out the horizontal splitting test for all of the even electrodes concurrently. The split droplets get merged at the odd electrodes; therefore, the merging test is performed at the same time. Similarly, by carrying out the splitting test for all of the odd electrodes concurrently, we can easily complete the horizontal merging test for all of the even electrodes. Thus, we can carry out all of the horizontal tests (merging and splitting) in one row by using only two manipulation steps. Similarly, all of the vertical tests in one column can be completed in two manipulation steps.

Following the aforementioned observations, we propose a parallel procedure to carry out mixing and splitting tests efficiently. The steps of the procedure are as follows.

Step 1) Route large droplets to all of the odd electrodes in a row, as shown in Fig. 11(a).

Step 2) Carry out the splitting test for all of the odd electrodes concurrently (large droplets are now on even electrodes), as shown in Fig. 11(b).

Step 3) Carry out the splitting test for all of the even electrodes concurrently (large droplets are now on odd electrodes), as shown in (c).

Step 4) Route the droplets consecutively to a capacitive-sensing circuit for test readouts.

Step 5) Repeat the test procedure for the next row.

Step 6) Repeat Steps 1)-5) for the columns.

Note that in the aforementioned method, only one capacitive-sensing circuit is used; therefore, the hardware cost is significantly reduced. However, additional droplet routing steps are 


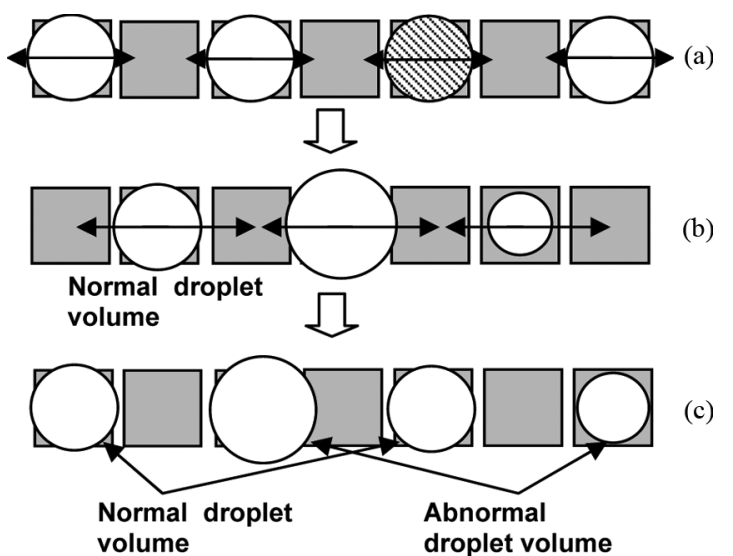

Fig. 11. Parallel mixing and splitting test for a row of electrodes (fault detection).

needed. In order to minimize the number of droplet manipulations, test results are read out not directly after each splitting or merging test, but after both of them are carried out as shown in the steps just shown. As a tradeoff, a more complicated test-result interpretation scheme is required.

If all of the tests in one row are executed without the detection of a malfunction, the droplet volume should be almost the same. However, if a malfunction occurs, volume variation is expected, as shown in Fig. 11.

In Fig. 11, the shaded droplet undergoes an unbalanced split during the splitting test. Since all other droplets are split evenly, this malfunction results in a pair of test droplets of abnormal volume - one bigger and the other smaller. If the next step of the test does not yield any malfunction, the droplet volume variation is propagated one electrode away. Therefore, we can easily detect the malfunction by checking the test results.

The proposed test method achieves high efficiency. An implicit assumption here is that adjacent electrodes are not defective. These defects can be detected by a separate structural test [33]. For an $N \times N$ array, only $N^{2}+N$ manipulation steps are needed, while the test method in prior work [33] requires $4 N^{2}$ steps. Moreover, the method uses only one capacitive-sensing circuit, irrespective of the array size. This is in contrast to [33], which requires $N^{2}$ array size. This is in contrast to [33], which requires $N^{2}$ capacitive-sensing circuits for an $N \times N$ microfluidic array. The potential reduction in production cost is therefore significant.

\section{ApPlication to Pin-Constrained ChIP}

In the discussion of the functional test method in Section V, we have assumed that the chip is controlled by using the direct-addressing method. In this section, we investigate the application of the functional test method to pin-constrained biochips. Four different pin-constrained biochip prototypes designed by using the techniques mentioned in Section III are discussed.

\section{A. N-Phase Chip}

In the $n$-phase chip, every $n$th electrode is electrically connected and controlled by using a single control pin. Therefore, every $n$ thth electrode must be activated/deactivated simultaneously, as shown in Fig. 12. Due to this constraint, the mixing and

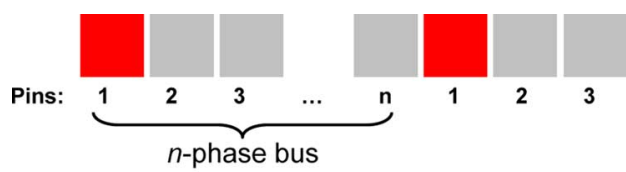

(a)

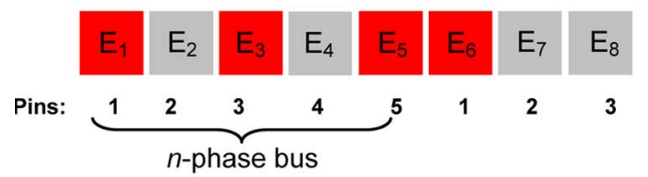

(b)

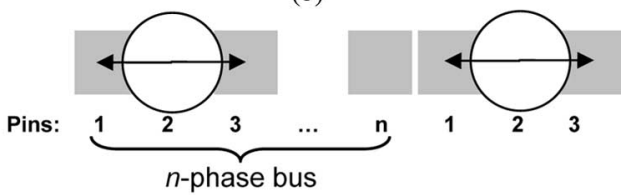

(c)

Fig. 12. Functional test on an $n$-phase bus chip.

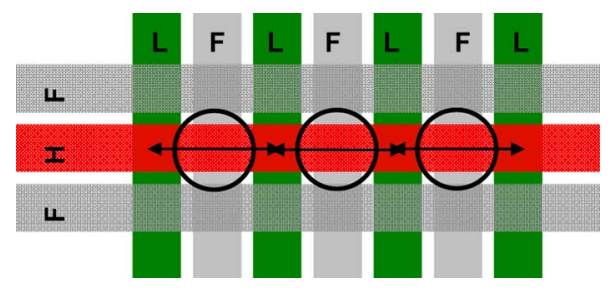

Fig. 13. Functional test on the cross-referencing-based chip.

splitting test cannot be carried out currently on every other electrode following the six steps presented in Section V-C. In the example of Fig. 12(b), a linear array consisting of eight electrodes is controlled by using a five-phase bus. To carry out splitting test on every other electrode (e.g., $\mathrm{E}_{1}, \mathrm{E}_{3}$, and $\mathrm{E}_{5}$ ), control pins 1 , 3 , and 5 need to be activated currently to hold the test droplets on these three electrodes. However, since electrode $\mathrm{E}_{6}$ is connected to Pin 1, it is also activated. This causes unintentional splitting of the test droplet on $\mathrm{E}_{5}$.

To avoid this problem, the proposed functional test procedure needs to be modified. For a microfluidic array controlled by using an $n$-phase bus, current mixing and splitting tests are only allowed on every $n$th electrode. Since these electrodes are connected to the same control pins, carrying out the splitting or mixing test on these electrodes requires the same pattern of pin activations. As shown in Fig. 12(c), executing a splitting test on $\mathrm{E}_{2}$ and $\mathrm{E}_{7}$ requires Pins 1 and 3 to be activated and Pin 2 to be deactivated. Therefore, carrying out the splitting/mixing test on these electrodes concurrently will not lead to unintentional fluidic operations.

\section{B. Cross-Referencing-Based Chip}

The proposed functional test method can also be applied to cross-referencing-based chips. The test procedure is the same as the one described in Section V-C. As shown in Fig. 13, to execute the splitting test for all of the even electrodes in the second row, test droplets are first routed to all of the odd electrodes. Next, the second row pin and all of the odd column pins are activated. This also completes the mixing test for all of the odd electrodes. In the final step, the row pin and all even column 


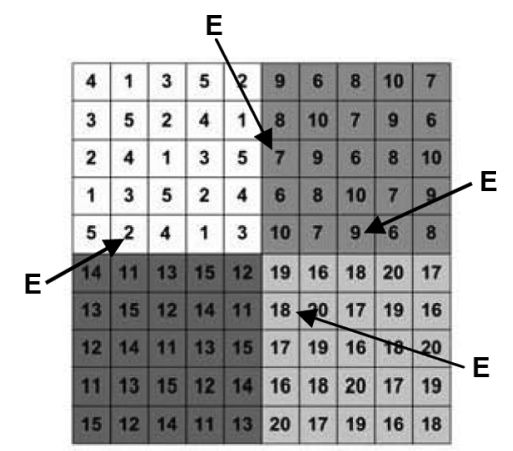

: Partitiop 1 (pins 1-5) $\square$ : Partitiop 2 (pins 6-10)

Partitiop 3 (pins 11-15) $\square:$ Partitiop 4 (pins 16-20)

Fig. 14. Functional test on array-partitioning-based chip.

pins are activated, thereby completing the splitting test for all of the odd electrodes and mixing test for the even electrodes.

\section{Array-Partitioning-Based Chip}

In [18], a biochip array is divided into several partitions based on the droplet-routing results. In each partition, the "Connect-5" pin-assignment algorithm is used to control the electrodes in it using five pins, as shown in Fig. 14. This pin-assignment algorithm allows free movement of a single droplet in the partition. However, only one droplet movement is allowed. Concurrent manipulation of more than two droplets within a single partition will lead to unintentional operations [18]. Therefore, no operations that involve two or more droplets (e.g., mixing and splitting) can be carried out within a single partition. In fact, these operations can only be executed by using the electrodes on the boundary of two partitions. Therefore, only the electrodes on the boundary need to be tested for these malfunctions.

Note that since only one droplet is allowed in each partition, while a mixing or splitting test is carried out on one electrode (e.g., $E_{1}$ ) on the boundary of two partitions (Partition 2 and Partition 4) in Fig. 14, no other electrodes in the same partition (e.g., $\mathrm{E}_{3}$ ) can be tested at the same time. As a result, boundary electrodes can only be tested one by one and the concurrency of the functional test is compromised. Fortunately, the total length of the boundaries on a chip is bounded by $2 \times N$ for an $N \times$ $N$ array. Only a limited number of iterations of functional test are needed. Moreover, it can been seen from Fig. 14 that the functional test of electrodes on different boundaries of different partitions (e.g., $E_{1}$ on the boundary of partition 2 and partition 4 and $E_{3}$ on the boundary of partition 1 and partition 3) uses completely different sets of control pins. Thus, these tests can be carried out concurrently. This increases the parallelism of the functional test. Moreover, a higher test frequency (i.e., the droplet activation rate) can be used to shorten the test time.

\section{Broadcast-Addressing-Based Chip}

Next, we apply the functional test method to a broadcast-addressing-based chip. Due to the constraints introduced by the pin-assignment results, not all of the cells on the chip can be tested. For example, the mixing and splitting test cannot be applied in the highlighted area in Fig. 15(b). To mix two droplets

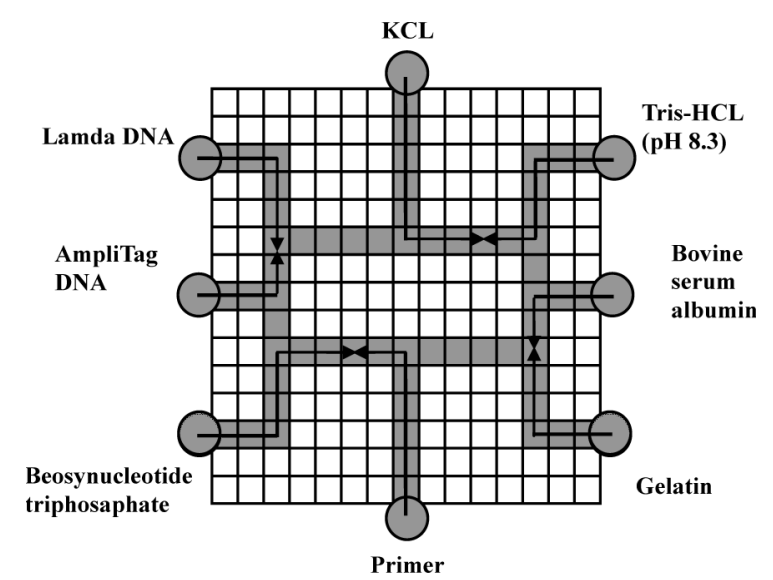

(a)

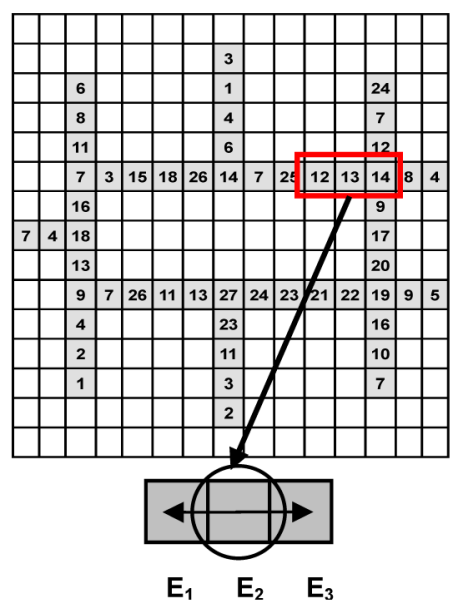

(b)

Fig. 15. Functional test for the broadcast-addressing-based chip. (a) Layout and droplet routes for the multiplexed-assay chip. (b) Broadcast-addressingbased pin-assignment result.

seated on $\mathrm{E}_{1}$ and $\mathrm{E}_{3}$ at the electrode $\mathrm{E}_{2}$, controlled by highlighting control pin 13 , the electrode above $\mathrm{E}_{3}$ will also be activated while we move the droplet from $\mathrm{E}_{3}$ to $\mathrm{E}_{1}$ and split it.

However, a broadcast-addressing-based chip is designed to execute a predetermined set of known bioassays [19]. We know exactly where the mixing and splitting operations will be carried out. So there is no need to test for malfunctions on other cells on the chip. Since the number of electrodes to be tested is very limited, only a small number of test steps are needed. Again, a higher test frequency can be used to reduce test time. To further increase concurrence, we can use the algorithm presented in [34] to check the compatibility of droplet movements during the functional test.

\section{EXPERIMENTAL AND SIMULATION RESULTS}

In this section, we apply the proposed functional test methods to a fabricated chip. The chip-under-test is a printed-circuitboard (PCB) microfluidic platform for the polymerase chain reaction (PCR), as shown in Fig. 16. PCR is a widely used procedure in DNA analysis for rapid enzymatic amplification of specific DNA strands. The platform consists of two columns and 


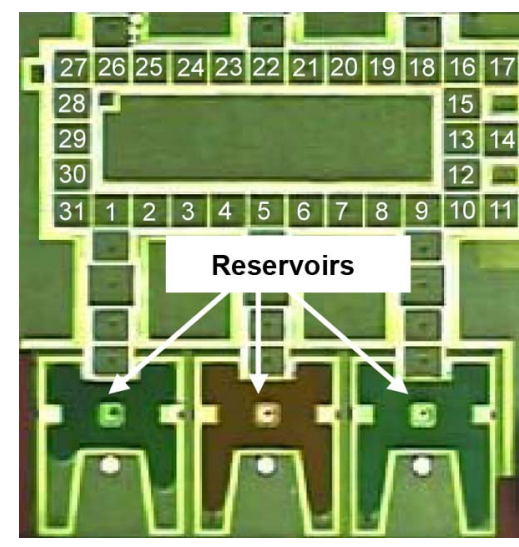

Fig. 16. Fabricated biochip for PCR.

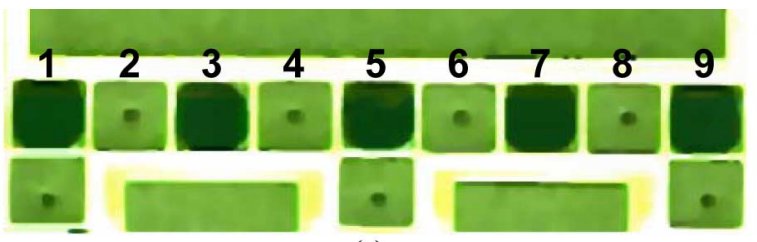

(a)

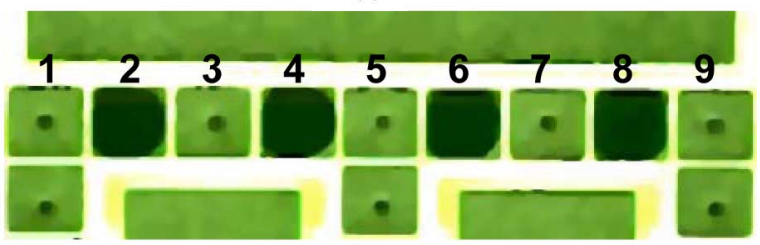

(b)

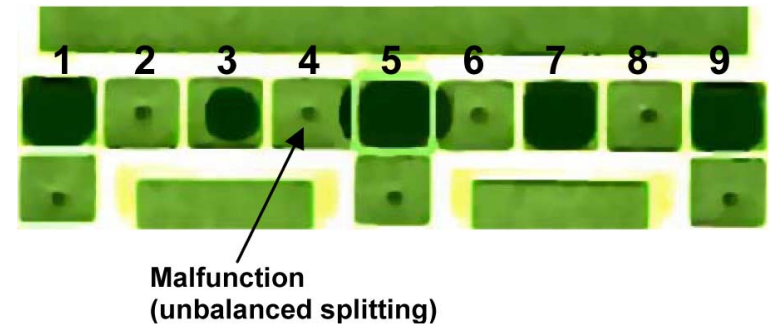

(c)

Fig. 17. Mixing and splitting test for a fabricated PCR chip (bottom row of Fig. 16).

two rows of electrodes, three reservoirs, and routing electrodes that connect the reservoirs to the array.

We applied the proposed functional test methods to this chip. The dispensing test and routing test are trivial due to the simple structure of the chip. Therefore, we only focused on the mixing and splitting tests. Following the steps in Section V-C, we first targeted the bottom row and dispensed five test droplets to the odd electrodes, as shown in Fig. 17(a).

Then, the splitting test of the even electrodes was carried out. Droplets were split and merged on the even electrodes. In Fig. 17(b), we see a series of droplets of the same volume resting on the even electrodes, which means that all of the odd electrodes passed the splitting test, and merging at the even electrodes worked well. However, when we carried out the splitting test on the even electrodes, a large variation in droplet volume was observed on the third and fifth electrodes; see Fig. 17(c). This variation implied a malfunction, leading to unbalanced splitting on the fourth electrode. The malfunction was detected when the droplets were routed to the capacitive-sensing circuit. We then labeled the fourth electrode on the bottom row as an unqualified splitting site so that synthesis tools will not map a splitter to it. Thus, the system robustness of the synthesized design was enhanced.

We next evaluate the improvement in system robustness using a biochip for a real-life protein assay [35]. Based on the Bradford reaction [35], the protocol for a generic droplet-based colorimetric protein assay is as follows. First, a droplet of the sample, such as serum or some other physiological fluid containing protein, is generated and dispensed into the biochip. Buffer droplets, such as $1 \mathrm{M} \mathrm{NaOH}$ solution, are then introduced to dilute the sample to obtain a desired dilution factor $(D F)$. This on-chip dilution is performed by using multiple hierarchies of binary mixing/splitting phases, referred to as the interpolating serial dilution method [3]. The mixing of a sample droplet of protein concentration $C$ and a unit buffer droplet results in a droplet with twice the unit volume, and concentration $C / 2$. Splitting this large droplet results in two unit-volume droplets of concentration $C / 2$ each. Continuing this step in a recursive manner using diluted droplets as samples, an exponential dilution factor of $D F=2^{N}$ can be obtained in $N$ steps. After dilution, droplets of reagents, such as Coomassie brilliant blue G-250 dye, are dispensed into the chip, and they mix with the diluted sample droplets. Next, the mixed droplet is transported to a transparent electrode, where an optical detector (e.g., a light-emitting diode (LED) photodiode setup) is integrated. The protein concentration can be measured from the absorbance of the products of this colorimetric reaction by using a rate kinetic method [32]. Finally, after the assay is completed, all droplets are transported from the array to the waste reservoir. A sequencing graph model can be developed from the aforementioned protocol for a protein assay $(D F=128)$, as shown in Fig. 18. There are a total of 103 nodes in one-to-one correspondence with the set of operations in a protein assay, where $D s S, D s B_{i}(i=1, \ldots, 39)$, and $D s R_{i}(i=1, \ldots, 8)$ represent the generation and dispensing of the sample, buffer, and reagent droplets, respectively.

In addition, $\operatorname{Dlt}_{i}(i=1, \ldots, 39)$ denotes the binary dilution (including mixing/splitting) operations, $\operatorname{Mix}_{i}(i=1, \ldots, 8)$ represents the mixing of diluted sample droplets and reagent droplets; and ${ } t_{i}(i=1, \ldots, 8)$ denotes the optical detection of the droplets. Until the fourth step of a serial dilution, all diluted sample droplets are retained in the microfluidic array. After that stage, for each binary dilution step, only one diluted sample droplet is retained after splitting, while the other droplet is moved to the waste reservoir.

The basic operations for protein assay have been implemented on a digital microfluidic biochip [3], [34]. Experiments indicate that the dispensing operation takes $7 \mathrm{~s}$ [3]. The operation times of various mixers have been found to be different [3]. A binary dilution operation can also be easily implemented by mixing the sample droplet followed by droplet splitting. The absorbance of the assay product can be measured by using an integrated LED-photodiode setup. Experiments indicate this absorbance measurement takes $30 \mathrm{~s}$ [34]. The microfluidic 


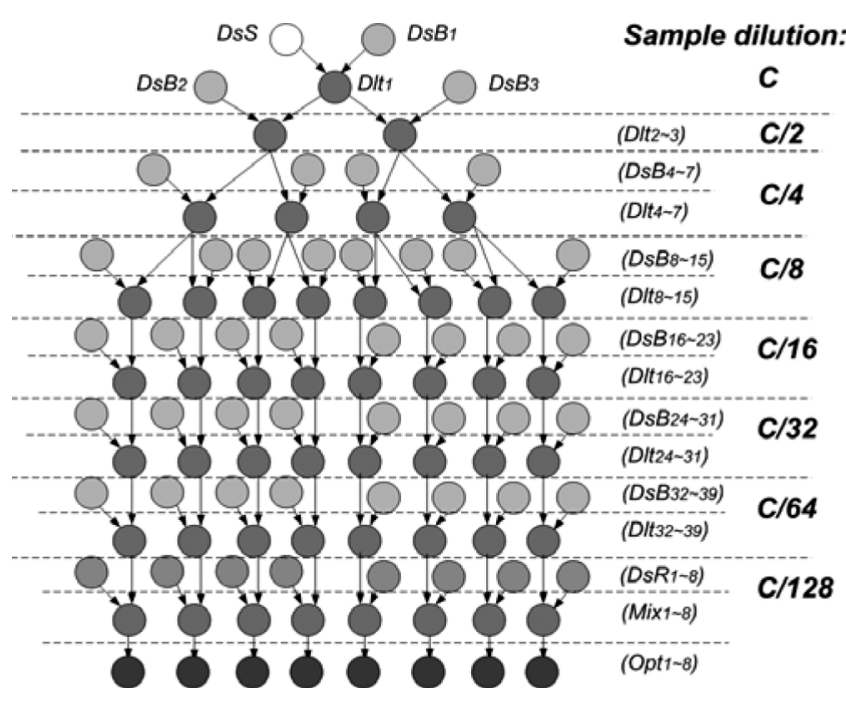

Fig. 18. Sequencing graph for a protein assay.

TABLE II

EXPERIMENTALlY CHARACTERIZED MODULE LIBRARY FOR SYNTHESIS

\begin{tabular}{|l|l|c|}
\hline Operation & Resource & Time (s) \\
\hline DsS;DsB; DsR & On-chip reservoir/dispensing port & 7 \\
\hline \multirow{4}{*}{ Dlt } & 2x2-array dilutor & 12 \\
\cline { 2 - 3 } & 2x3-array dilutor & 8 \\
\cline { 2 - 3 } & 2x4-array dilutor & 5 \\
\cline { 2 - 3 } & 4-electrode linear array dilutor & 7 \\
\hline \multirow{4}{*}{ Mix } & 2x2-array mixer & 10 \\
\cline { 2 - 3 } & 2x3-array mixer & 6 \\
\cline { 2 - 3 } & 2x4-array mixer & 3 \\
\cline { 2 - 3 } & 4-electrode linear array mixer & 5 \\
\hline Opt & LED+Photodiode & 30 \\
\hline Storage & Single cell & N/A \\
\hline
\end{tabular}

module library for a protein assay is shown in Table II. A $10 \times 10$ microfluidic array is used to execute the assay.

Next, we evaluate system dependability of the synthesized design for three cases: 1) no testing is carried out; 2) only the structural test is carried out; and 3) the functional test is carried out. A design is deemed to fail if any module suffers from a defect or a malfunction (e.g., a mixer suffering from a faulty mixing site).

We generate 200 simulated samples of faulty arrays. Each faulty array is derived by randomly injecting a fault (due to defects and malfunctions) in the array. Note that we do not specify the types of injected malfunctions. Here, we simply assume that all injected malfunctions can be detected by the proposed functional test and cannot be detected through the structural test. Let A be the event that a unit cell has a defect. We let the defect probability $p=\mathrm{P}[\mathrm{A}]$ take two values, namely 0.01 and 0.05 . Let $\mathrm{B}$ be the event that a group of electrodes suffers from a malfunction. Since a defective unit cell leads to a malfunction of the module where it is used, we are interested here in the conditional probability $q=\mathrm{P}[\mathrm{B} \mid \overline{\mathrm{A}}]$ (i.e., the probability that a module is malfunctioning even if there is no defect in it). We consider four values of $q$, namely, 0.02, 0.04, 0.06, and 0.08. For each faulty array, the structural test method from [13] is carried out to detect and locate defect sites.

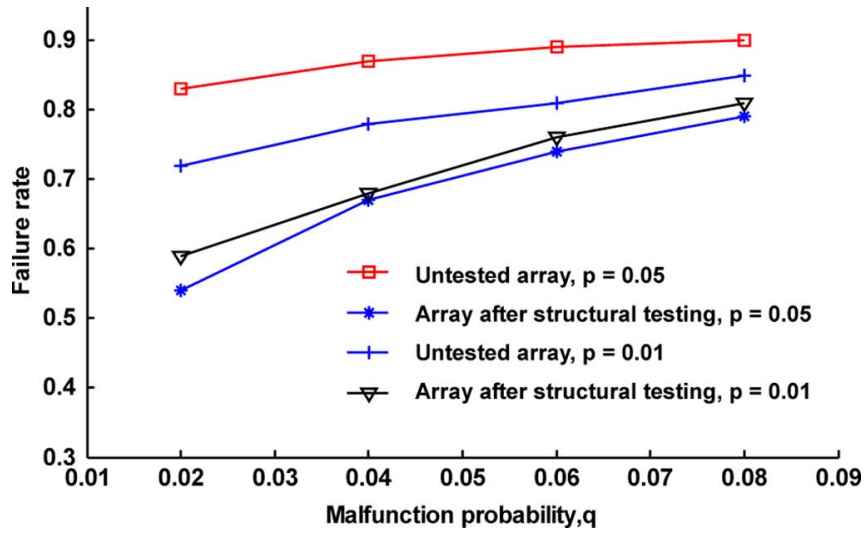

Fig. 19. Failure rate for synthesized design without testing and with structural testing.

Next, the synthesis method from [14] is used to map the protein array on to defect-free regions of the array. We also use the functional test to detect and locate malfunctions in the array. These malfunctions are then bypassed during the synthesis of the chip for the protein array. As a baseline, we also carry out the synthesis for an array to which neither the structural test nor the functional test have been applied.

First, we determine the failure rate $R, 0 \leq R \leq 1$, for the three scenarios when the protein assay is mapped to an array with defects and malfunctions. When functional testing is carried out, the failure rate due to target defects and malfunctions is zero because all of them are detected by the test procedure. If no testing is carried out, the failure rate is as high as 0.85 (i.e., the protein assay fails for as much as $85 \%$ of the 200 simulated chips). If structural testing is used, the failure rate is lower, but it is still significant-as high as 0.75 .

Fig. 19 shows that as the malfunction probability increases, the failure rate $R$ becomes considerable even when structural testing is used. Moreover, the benefits of structural testing are less evident for the smaller value of the defect probability (i.e., $p=0.01$ ). Therefore, functional testing is needed to augment droplet-transportation-based structural testing for digital microfluidic arrays. A counterintuitive finding from Fig. 19 is that the failure rate is lower for $p=0.05$ compared to $p=0.01$. This occurs because large $p$ implies that there is a low likelihood of a defect-free cell. Hence, structural testing is likely to catch such defects.

The detection of more defects and malfunctions using functional testing leads to a corresponding increase in the assay completion time. This occurs because fault detection and fault location leave fewer unit cells available for the protein assay, and the synthesis procedure employs less parallelism in the execution of the microfluidic operation. Fig. 20 shows the assay completion time for the three scenarios that we are considering for the protein assay. The completion times shown in Fig. 20 are the average values (arithmetic mean) over 200 simulation runs. As expected, the bioassay time is slightly higher when functional testing is used, and the increase is more for higher values of the malfunction probability $q$. This increase is acceptable because functional testing ensures that the assay will run to completion 


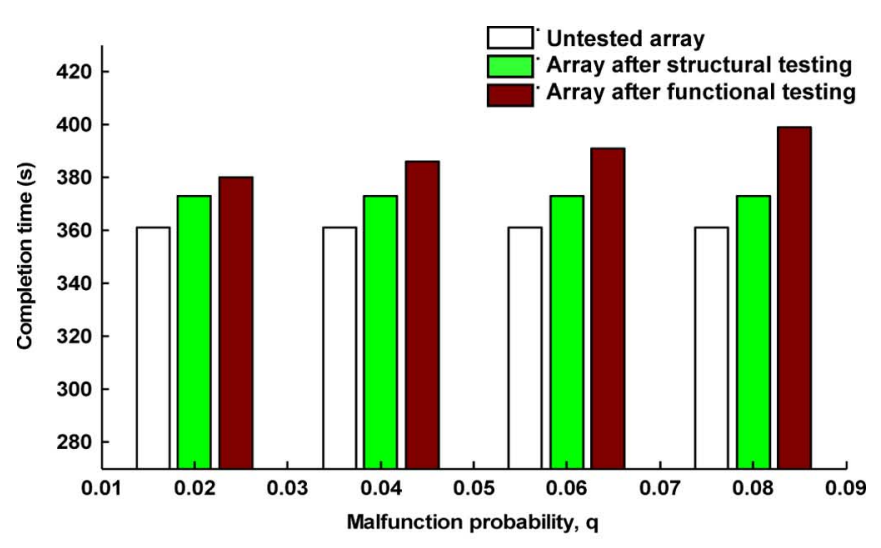

(a)

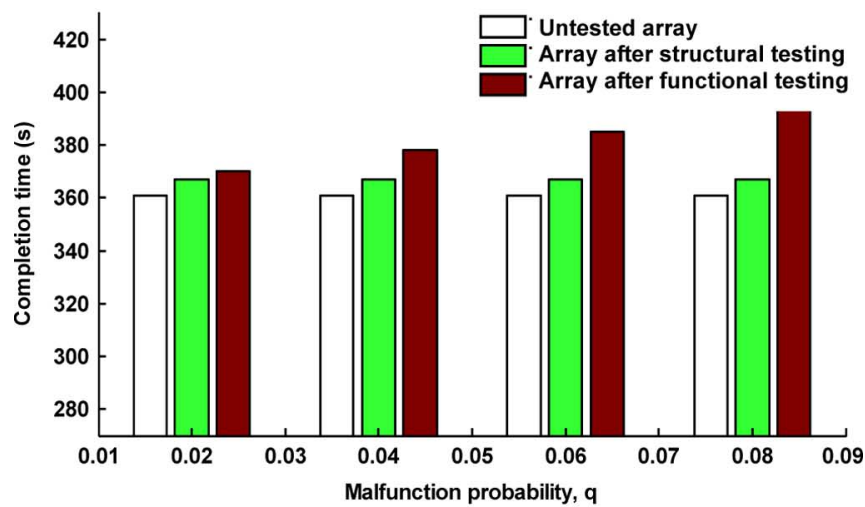

(b)

Fig. 20. Bioassay completion time for synthesized design without test, with structural test, and with functional test for defect occurrence probability of (a) $\mathrm{P}[\mathrm{A}]=0.01$. (b) $\mathrm{P}[\mathrm{A}]=0.05$.

if the fluidic operations are mapped only on qualified regions of the array.

Next, we evaluate the functional test scheme on pin-constrained arrays. For each pin-constrained design method presented in Section VI, we choose one chip design prototype for discussion, as shown in Fig. 21. Fig. 21(a) shows a linear chip used for the on-chip dilution. The chip is addressed by using a five-phase bus. Fig. 21(b) presents a $10 \times 10$ array for a multiplexed bioassay consisting of a glucose assay and a lactate assay based on colorimetric enzymatic reactions. These assays have been demonstrated recently [16]. For each sample or reagent, two droplets are dispensed into the array. Four pairs of droplets (i.e., $\left\{S_{1}, R_{1}\right\},\left\{S_{1}, R_{2}\right\},\left\{S_{2}, R_{1}\right\}$, and $\left\{S_{2}, R_{2}\right\}$ ) are routed together in sequence for the mixing operation. Mixed droplets are finally routed to the detection site for analysis. Fig. 21(c) provides an array-partitioning-based chip design for the multiplexed assay. The chip is divided into four partitions, each partition is controlled by using a dedicate set of pins. Fig. 21(d) shows a cross-referencing-based chip design for the same assay. The simple design prototype is composed of ten row pins and ten column pins. Finally, for the broadcast-address method, the design shown in Fig. 15 is used. The chip is designed for executing on-chip PCR reactions.

Next, we apply the extended functional test method proposed in Section VI to these pin-constrained chips. The number of

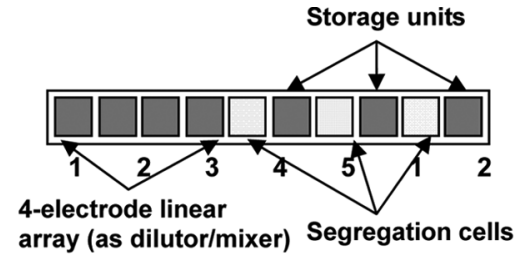

(a)

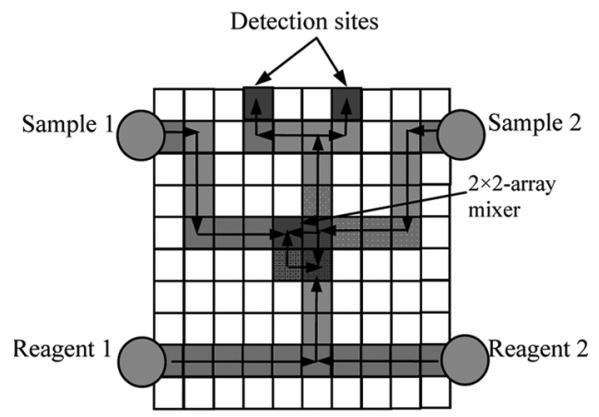

(b)

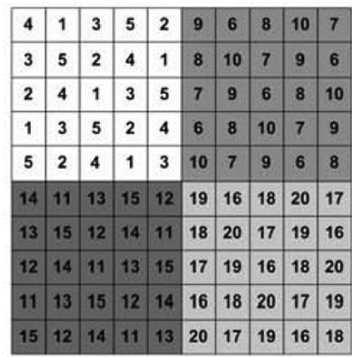

Partition 1 (pins 1-5) $\quad \square$ : Partition 2 (pins 6-10)

Partition 3 (pins 11-15) $\square$ : Partition 4 (pins 16-20)

(c)

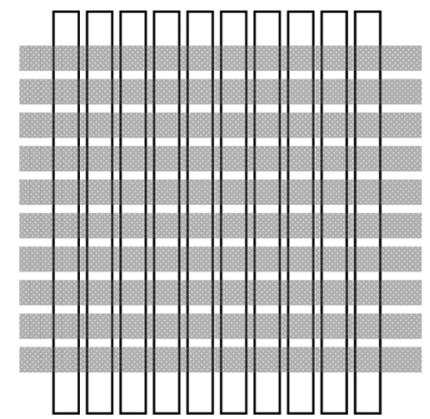

(d)

Fig. 21. Pin-constrained chip designs for functional test evaluation. (a) Linear five-phase-bus chip for dilution. (b) Layout and droplet routes for the multiplexed-assay chip. (c) Array-partitioning-based array for the multiplexed assay. (d) Cross-referencing-based array for multiplexed assay.

droplet manipulation steps are recorded and shown in Fig. 22. Fig. 22 also presents the number of manipulation steps required by the functional test assuming the chip is direct-addressable. The functional test on a cross-referencing-based chip requires the same test time as for a direct-addressable chip. For other pin-constrained designs, the function test can still be carried out effectively, though with an increase in test application time. Considering the significant reduction in the number of control pins, this compromise is acceptable. Moreover, the reduction in test concurrency can be avoided by increasing the test frequency. 


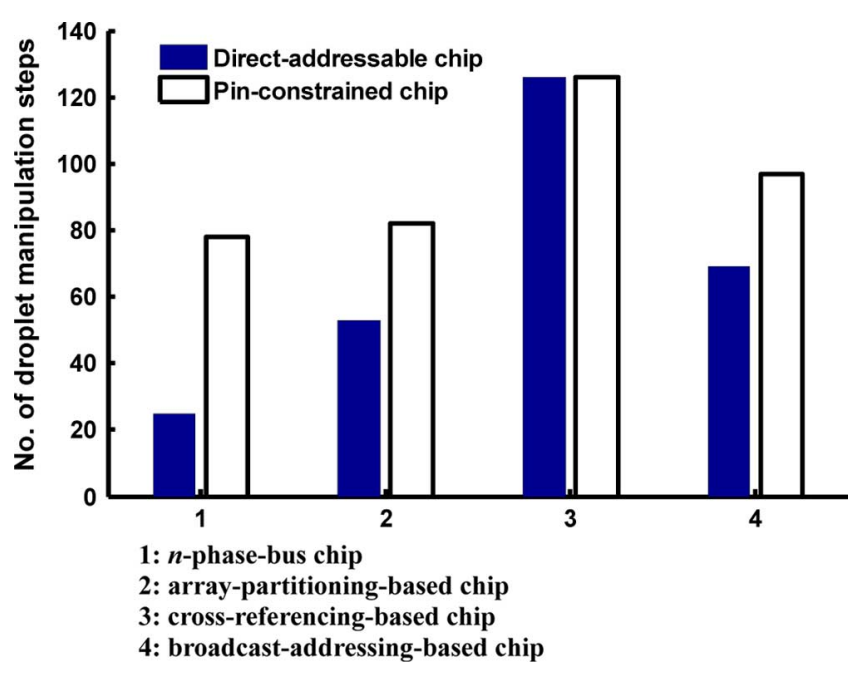

Fig. 22. Number of droplet-manipulation steps for the functional test of directaddressable and pin-constrained chips.

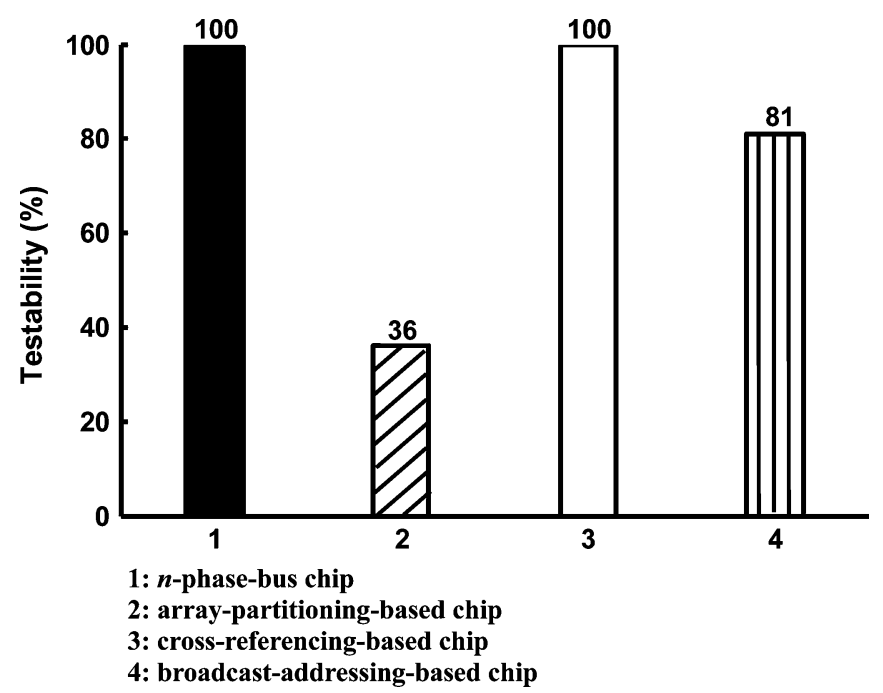

Fig. 23. Functional testability of pin-constrained chips.

Another important critical quality to measure the extended functional test method for pin-constrained designs is testability. As mentioned in Section VI, due to the connection constraints, not all of the electrodes on a pin-constrained array can be tested. Therefore, we define testability as the ratio of the number of testable electrodes over the total number of electrodes in the array. High testability indicates that the test method can probe the functionality of the chip thoroughly and identify a large number of qualified regions for a target application, which, in turn, contributes to increased flexibility for design and fault tolerance. Here, we calculate testability for the four pin-constrained designs. Results are shown in Fig. 23.

Fig. 23 shows that the proposed functional test method achieves high testability $(>80 \%)$ on the $n$-phase chip, the cross-referencing-based array, and the broadcast-addressing-based array. The testability for the array-partitioning-based chip appears to be low. This is due to the fact that on an array-partitioning-based chip, the mixing and splitting operations are only allowed on the boundary cells. If we take this restriction into account, the testability is as high as $100 \%$.

\section{CONCLUSION}

We have presented several techniques for the functional testing of droplet-based microfluidic biochips. These techniques address fundamental biochip operations, such as droplet dispensing, droplet transportation, mixing, splitting, and capacitive sensing. Functional testing is carried out by using parallel droplet pathways, and it leads to qualified regions where synthesis tools can map microfluidic functional modules. We have demonstrated functional testing for a fabricated biochip that is used for PCR. We have also presented simulation results for a protein assay, and quantified the small increase in assay completion time that is needed to achieve $100 \%$ coverage of the target defects and malfunctions with functional testing. Finally, we described the application of these functional test methods to pin-constrained chips.

\section{REFERENCES}

[1] R. B. Fair, A. Khlystov, T. D. Tailor, V. Ivanov, R. D. Evans, P. B. Griffin, V. Srinivasan, V. K. Pamula, M. G. Pollack, and J. Zhou, "Chemical and biological applications of digital-microfluidic devices," IEEE Design Test Comput., vol. 24, no. 1, pp. 10-24, Jan./Feb. 2007.

[2] R. B. Fair, "Digital microfluidics: is a true lab-on-a-chip possible?", Microfluidics Nanofluidics, vol. 3, pp. 245-281, 2007.

[3] R. B. Fair, V. Srinivasan, H. Ren, P. Paik, V. K. Pamula, and M. G. Pollack, "Electrowetting-based on-chip sample processing for integrated microfluidics," in Proc. IEEE Int. Electron Devices Meet., 2003, pp. 32.5.1-32.5.4.

[4] E. Verpoorte and N. F. De Rooij, "Microfluidics meets MEMS," Proc. IEEE, vol. 91, no. 6, pp. 930-953, Jun. 2003.

[5] J.Zeng and T. Korsmeyer, "Principles of droplet electrohydrodynamics for lab-on-a-chip," Lab on a Chip, vol. 4, pp. 265-277, 2004.

[6] J. F. Zhong, Y. Chen, J. S. Marcus, A. Scherer, S. R. Quake, C. R. Taylor, and L. P. Weiner, "A microfluidic processor for gene expression profiling of single human embryonic stem cells," Lab on a Chip, vol. 8, pp. 68-74, 2008.

[7] X. Cheng, Y. Liu, D. Irimia, U. Demirci, L. Yang, L. Zamir, W. Rodriguez, M. Toner, and R. Bashir, "Cell detection and counting through cell lysate impedance spectroscopy in microfluidic devices," Lab on a Chip, vol. 7, pp. 746-755, 2007.

[8] P. Garstecki, I. Gitlin, W. Diluzio, E. Kumacheva, H. A. Stone, and G. M. Whitesides, "Formation of monodisperse bubbles in a microfluidic flow-focusing device," Appl. Phys. Lett., vol. 85, pp. 2649-2651, 2004.

[9] R. B. M. Schasfoort, S. Schlautmann, J. Hendrikse, and A. van den Berg, "Field-effect flow control for microfabricated fluidic networks," Science, vol. 286, pp. 942-945, 1999.

[10] B. M. Paegel, R. G. Blazej, and R. A. Mathies, "Microfluidic devices for DNA sequencing: Sample preparation and electrophoretic analysis," Current Opin. Biotechnol., vol. 14, pp. 42-50, 2003.

[11] T. Xu, P. Thwar, V. Srinivasan, V. K. Pamula, and K. Chakrabarty, "Digital microfluidic biochip for protein crystallization," presented at the IEEE-NIH Life Science Systems and Applications Workshop, Bethesda, MD, 2007.

[12] Silicon Biosystems. [Online]. Available: http://www.siliconbiosystems.com/DEPArray.page

[13] F. Su, S. Ozev, and K. Chakrabarty, "Testing of droplet-based microelectrofluidic systems," in Proc. Int. Test Conf., 2003, pp. 1192-1200.

[14] F. Su and K. Chakrabarty, "Unified high-level synthesis and module placement for defect-tolerant microfluidic biochips," in Proc. IEEE/ACM Design Automation Conf., 2005, pp. 825-830.

[15] P. Y. Paik, V. K. Pamula, M. G. Pollack, and K. Chakrabarty, "Coplanar digital microfluidics using standard printed circuit board processes," presented at the MicroTAS, Boston, MA, 2005.

[16] V. Srinivasan, V. K. Pamula, and R. B. Fair, "An integrated digital microfluidic lab-on-a-chip for clinical diagnostics on human physiological fluids," Lab on a Chip, pp. 310-315, 2004.

[17] S. K. Fan, C. Hashi, and C. J. Kim, "Manipulation of multiple droplets on $\mathrm{N} \times \mathrm{M}$ grid by cross-reference EWOD driving scheme andpressurecontact packaging," in Proc. IEEE MEMS Conf., 2003, pp. 694-697. 
[18] T. Xu, W. Hwang, F. Su, and K. Chakrabarty, "Automated design of pin-constrained digital microfluidic biochips under droplet-interference constraints," ACM J. Emerging Technol. Comput. Syst., vol. 3, 2007.

[19] T. Xu and K. Chakrabarty, "Broadcast electrode-addressing for pin-constrained multi-functional digital microfluidic biochips," in Proc. IEEE/ACM Design Automation Conf., 2008, pp. 173-178.

[20] N. Deb and R. D. Blanton, "Analysis of failure sources in surface-micromachined MEMS," in Proc. Int. Test Conf., 2000, pp. 739-749.

[21] A. Dhayni, S. Mir, L. Rufer, and A. Bounceur, "Pseudorandom functional BIST for linear and nonlinear MEMS," in Proc. Design Automation Test Eur., 2006, pp. 664-669.

[22] T. Xu and K. Chakrabarty, "Parallel scan-like test and multiple-defect diagnosis for digital microfluidic biochips," IEEE Trans. Biomed. Circuits Syst., vol. 1, no. 2, pp. 148-158, Jun. 2007.

[23] H. G. Kerkhoff, "Testing microelectronic biofluidic systems," IEEE Design Test Comput., vol. 24, no. 1, pp. 72-82, Jan./Feb. 2007.

[24] F. Su, S. Ozev, and K. Chakrabarty, "Ensuring the operational health of droplet-based microelectrofluidic biosensor systems," IEEE J. Sens., vol. 5, no. 4, pp. 763-773, Aug. 2005.

[25] T. H. Schulte, R. L. Bardell, and B. H. Weigl, "Microfluidic technologies in clinical diagnostics," Clini. Chim. Acta, vol. 321, pp. 1-10, 2002.

[26] F. Su, S. Ozev, and K. Chakrabarty, "Concurrent testing of droplet-based microfluidic systems for multiplexed biomedical assays," in Proc. Int. Test Conf., 2004, pp. 883-892.

[27] F. Su and K. Chakrabarty, "Defect-oriented testing and diagnosis of digital microfluidics-based biochips," in Proc. IEEE Int. Test Conf., 2005, pp. 487-496.

[28] V. Hlady, R. A. Wagenen, and J. D. Andrade, Surface and Interfacial Aspects of Biomedical Polymers: Protein Adsorption, J. D. Andrade, Ed. New York: Plenum, 1985, vol. 2, p. 81.

[29] M. G. Pollack, "Electrowetting-based microactuation of droplets for digital microfluidics," Ph.D dissertation, Duke University, Durham, NC, 2001.

[30] M. G. Pollack, P. Y. Paik, A. D. Shenderov, V. K. Pamula, F. S. Dietrich, and R. B. Fair, "Investigation of electrowetting-based microfluidics for real-time PCR applications," presented at the Int. Conf. Micro Total Analysis Systems (uTAS), 2003.

[31] P. Paik, V. K. Pamula, M. G. Pollack, and R. B. Fair, "Rapid droplet mixers for digital microfluidic systems," Lab on a Chip, vol. 3, pp. 253-259, 2003.

[32] P. Paik, V. K. Pamula, M. G. Pollack, and R. B. Fair, "Electrowettingbased droplet mixers for microfluidic systems," Lab on a Chip, vol. 3, pp. 28-33, 2003.

[33] F. Su, W. Hwang, A. Mukherjee, and K. Chakrabarty, "Testing and diagnosis of realistic defects in digital microfluidic biochips," J. Electron. Testing: Theory Appl., vol. 23, pp. 219-233, 2007.

[34] E. J. Griffith, S. Akella, and M. K. Goldberg, "Performance characterization of a reconfigurable planar-array digital microfluidic system," IEEE Trans. Comput.-Aided Design Integr. Circuits Syst., vol. 25, no. 2, pp. 340-352, Feb. 2006.

[35] V. Srinivasan, V. K. Pamula, P. Paik, and R. B. Fair, "Protein stamping for MALDI mass spectrometry using an electrowetting-based microfluidic platform," Proc. SPIE, vol. 5591, pp. 26-32, 2004.

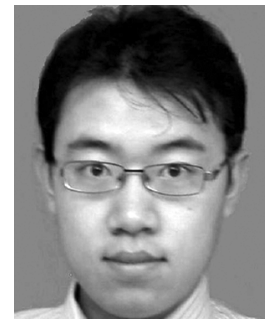

Tao Xu (S'09) received the B.E. degree in electrical engineering from Zhejiang University, Hangzhou, China, in 2005, and the M.S. degree and Ph.D. degrees in electrical and computer engineering from Duke University, Durham, NC, in 2007 and 2008, respectively.

Currently, he is a Senior DFT engineer at Cisco Systems, Morrisville, NC.

His research interests include design and testing of mixed-technology microsystems, electronic design automation, mixed-signal very large-scale integrated design, microelectromechanical-systems modeling and simulation. He has published 11 conference papers and six journal papers.

Dr. Xu was the Best Paper Award winner at the 20th International VLSI Conference.

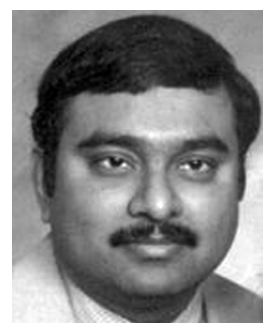

Krishnendu Chakrabarty (F08) received the B.Tech. degree from the Indian Institute of Technology, Kharagpur, in 1990, and the M.S.E. and $\mathrm{Ph} . \mathrm{D}$. degrees from the University of Michigan, Ann Arbor, in 1992 and 1995, respectively.

Currently, he is Professor with the Department of Electrical and Computer Engineering at Duke University, Durham, NC. He is also a Chair Professor in Software Theory at the School of Software, Tsinghua University, Beijing, China. His research projects include testing and design-for-testability of integrated circuits; digital microfluidics and biochips, circuits and systems based on DNA self-assembly, and wireless sensor networks. He has authored seven books on these topics, published many papers in journals and refereed conference proceedings, and has given more than 120 invited, keynote, and plenary talks.

Prof. Chakrabarty is a recipient of the National Science Foundation Early Faculty (CAREER) award, the Office of Naval Research Young Investigator Award, the Humboldt Research Fellowship from the Alexander von Humboldt Foundation, Germany, and several best papers awards at IEEE conferences. $\mathrm{He}$ is a Distinguished Engineer of ACM. He is a 2009 Fellow of the Japan Society for the Promotion of Science (JSPS) and a recipient of the 2008 Duke University Graduate School Dean's Award for excellence in mentoring. He served as a Distinguished Visitor of the IEEE Computer Society during 2005-2007, and as a Distinguished Lecturer of the IEEE Circuits and Systems Society during 2006-2007. Currently, he serves as an ACM Distinguished Speaker. He is an Associate Editor of the IEEE TRANSACTIONS ON COMPUTER-AIDED DESIGN OF INTEGRATED CIRCUITS AND SYSTEMS, IEEE TRANSACTIONS ON VERY LARGE SCALE INTEGRATED SYSTEMS, IEEE TRANSACTIONS ON BIOMEDICAL CIRCUITS AND SYSTEMS, and the ACM Journal on Emerging Technologies in Computing Systems. He is also Editor of IEEE DESIGN \& TEST OF COMPUTERS and the Journal of Electronic Testing: Theory and Applications. He is a Distinguished Engineer of ACM. 